\title{
Computation of 3D Induction Hardening Problems by Combined Finite and Boundary Element Methods
}

\author{
Sven Wanser, Laurent Krähenbühl, Alain Nicolas \\ CEGELY - C.N.R.S. URA n829 \\ Ecole Centrale de Lyon - 36, avenue Guy de Collongue - B.P. 163 - 69131 Ecully Cedex - France \\ Fax: (+33) 78.43.37.17 e-mail: wanser@trotek.ec-lyon.fr
}

\begin{abstract}
A method for the solution of high frequency three dimensional coupled magneto-thermal problems such as those of the induction hardening of steel is proposed. The magnetic problem is treated first using the Boundary Element Method (BEM) to calculate magnetic parameters. From eddy-currents and Joule effects, source values are obtained to solve the thermal problem by the Finite Element Method (FEM). The two problems are then indirectly coupled to obtain a solution for the entire problem.
\end{abstract}

\section{INTRODUCTION}

In the modelling of high frequency induction-hardening problems by the Finite Element Method, special efforts are made to have a fine mesh within the skin depth of the material. The higher the frequency, the finer the mesh required in the skin depth region and the more costly in terms of computation time and memory space. Existing automatic meshing processors have hitherto proved to be prohibitive in such costs. It is also a tedious task to undertake a manual meshing of a 3D problem.

It is for these reasons that we used the Boundary Element and Surface Impedance Methods implemented in the PHI3D software package developed at the Centre de Génie Electrique de Lyon (CEGELY) in France to solve the high frequency magnetodynamic problem. By transfering all electromagnetic phenomena to the surface of the component to be hardened, we obtain the distribution of eddy-currents and the surface power densities on the surfaces of the component. The surface power density is taken as the surface thermal source in the solution of the thermal problem using the software package FLUXEXPERT based on the Finite Element Method [1]. An FEM-based treatment of the thermal problem was chosen because we wish to study phenomena inside the material under consideration.

\section{FORMULATION}

\section{A. Magnetodynamics}

Maxwell's equations:

$$
\operatorname{curl} \mathbf{E}=-\frac{\partial \mathbf{B}}{\partial \mathrm{t}} \quad \operatorname{div} \mathbf{B}=\mathbf{0}
$$

$$
\begin{array}{lr}
\operatorname{curl} \mathbf{H}=\mathbf{J} & \operatorname{div} \mathbf{E}=\mathbf{0} \\
\mathbf{B}=\mu_{0} \mu_{\mathrm{r}} \mathbf{H} & \mathbf{J}=\sigma \mathbf{E}
\end{array}
$$

By separation of the variables we obtain the Reduced Scalar Potential in nonconducting regions:

$$
\begin{aligned}
& \mathbf{H}=\mathbf{H}_{0}-\mathbf{g r a d} \Omega \\
& \mathbf{H}_{0}: \text { magnetic field due to source currents, created without } \\
& \text { any influence of ferromagnetic material; } \\
& \text { grad } \Omega: \text { "material reaction" magnetic field, created by } \\
& \text { eddy-currents inside the ferromagnetic material; } \\
& \operatorname{div} \mathbf{B}=\operatorname{div} \mu_{0}\left(\mathbf{H}_{0}+\mathbf{H}_{\mathrm{m}}\right)=0 \\
& \Leftrightarrow \operatorname{div} \mu_{0} \mathbf{H}_{\mathrm{m}}=-\operatorname{div} \mu_{0} \operatorname{grad} \Omega=-\mu_{0} \Delta \Omega=0
\end{aligned}
$$

The governing equation of the Boundary Element Method:

$$
\begin{aligned}
& c \Omega(P)=\int_{\partial Q_{i}}\left[G(P, Q) \frac{\partial \Omega(Q)}{\partial n}-\Omega(Q) \frac{\partial G(P, Q)}{\partial n}\right] d S \\
& G: \text { Green's function; } \\
& \Omega: \text { scalar potential. }
\end{aligned}
$$

The problem is solved in the non-conducting region (air : $\sigma=0, \mu=1$ ) and the eddy-current phenomena inside the skin depth region are projected in a simple boundary condition. The skin depth $\delta$ is very thin compared with the dimensions of the component. The eddy-currents are assumed to flow parallel to the surface.

By combining Maxwell's equations (1) we obtain:

$$
\operatorname{curl}\left(\frac{1}{\sigma} \operatorname{curl} H\right)=-\mu \frac{\partial H}{\partial t}
$$

which leads to an exponential solution inside the metal [2]:

$$
\mathbf{H}(\mathrm{z})=\mathbf{H}_{\mathrm{s}} \exp \left[-\frac{(1+j) z}{\delta}\right]
$$




$$
\begin{aligned}
& \mathbf{j}(\mathrm{z})=\mathbf{j}_{\mathrm{s}} \exp \left[-\frac{(1+j) \mathbf{z}}{\delta}\right] \\
& \mathbf{H}_{\mathrm{s}}=\text { tangential magnetic field; } \\
& \mathbf{j}_{\mathrm{s}}=\text { surface current density } \\
& \mathbf{j}_{s}=\left[-\frac{(1+j)}{\delta}\right]\left(\mathbf{n} \times \mathbf{H}_{s}\right)
\end{aligned}
$$

From equations (6) and (9) we obtain the relationship between the surface current density and the normal component of the magnetic field [3], [4]:

$$
\text { n.curl } \mathbf{j}_{s}=\frac{(2 \mathrm{j} \mathbf{n} . \mathbf{H})}{\delta^{2}}=(1+\mathrm{j}) \operatorname{div}_{\mathrm{s}}\left(\frac{\mathbf{H}_{\mathrm{s}}}{\delta}\right)
$$

This leads to the relationship between the tangential $\mathbf{H}_{8}$ and normal $\mathbf{H}_{n}$ components of the magnetic field on the nonconducting side of the surface:

$$
\mathbf{H}_{\mathrm{n}}=\mathbf{H} \cdot \mathbf{n}=\frac{(1-j)}{2} \delta \mu_{\mathrm{r}} \operatorname{div}_{\mathrm{s}} \mathbf{H}_{\mathrm{s}}
$$

This is taken as a boundary condition when solving the magnetic problem in the air by PHI3D :

$$
\text { lap } \Omega=0
$$

The coupling with the thermal problem is effected by thermal sources, which are the eddy-currents heating the material. Using the surface impedance [2]

$$
\underline{z}=\frac{(1+j)}{\delta \sigma}
$$

we obtain

$$
\mathbf{E}=\underline{\mathbf{z}}\left(\mathbf{n} \times \mathbf{H}_{\mathbf{S}}\right)
$$

The surface power density is calculated by the Poynting vector $\mathbf{S}=\mathbf{E x H}$.

$$
\Rightarrow q_{w 0}=\frac{H^{2}}{\delta \sigma}
$$

Using this method PHI3D is able to treat either problems with solid inductors and problems with field concentrators. One can choose whether eddy-currents have to be calculated on the inductor-surface or not.

\section{B. Thermodynamics}

The general formulation of thermal conduction is:

$$
\gamma_{c_{p}} \frac{\partial T(x, t)}{\partial t}-\operatorname{div}[\lambda \operatorname{grad} T(x, t)]=W_{q}(x, t)
$$

where:

$$
\gamma \text { : mass density }\left(\mathrm{kg} \mathrm{m}^{-3}\right) \text {, }
$$

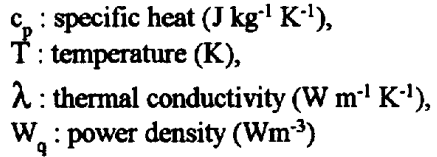

The boundary condition for heat convection and radiation is of the Fourier type:

$$
\begin{aligned}
& -\mathrm{q}_{\mathrm{w}}=\mathrm{h}\left(\mathrm{T}_{\mathrm{F}}-\mathrm{T}_{\mathrm{w}}\right)+\mathrm{s} \varepsilon\left(\mathrm{T}_{\mathrm{F}}^{4}-\mathrm{T}_{\mathrm{w}}{ }^{4}\right)+\mathrm{q}_{\mathrm{w} 0} \\
& \mathrm{~h}: \text { factor of heat-transmission }\left(\mathrm{W} \mathrm{m}^{-2} \mathrm{~K}^{-1}\right), \\
& \mathrm{s}: \text { Boltzmann constant, } \\
& \varepsilon \text { : emission coefficient, } \\
& \mathrm{T}_{\mathrm{w}}: \text { ambient temperature }(\mathrm{K}), \\
& \mathrm{T}_{\mathrm{F}} \text { : material temperature }(\mathrm{K}), \\
& \mathrm{q}_{\mathrm{w} 0} \text { : surface power density (heat flux). }
\end{aligned}
$$

\section{Coupling}

In induction heating the material characteristics are strongly interdependent. In magnetodynamics the electric conductivity at every point depends on the temperature. The magnetic permeability varies with the temperature and also with the magnetic field $\mathbf{H}$. In addition, all physical properties of the thermal problem are dependent on the temperature. The strong variation of the properties with the temperature makes the system non-linear. That is why the problems must be solved in a coupled manner.

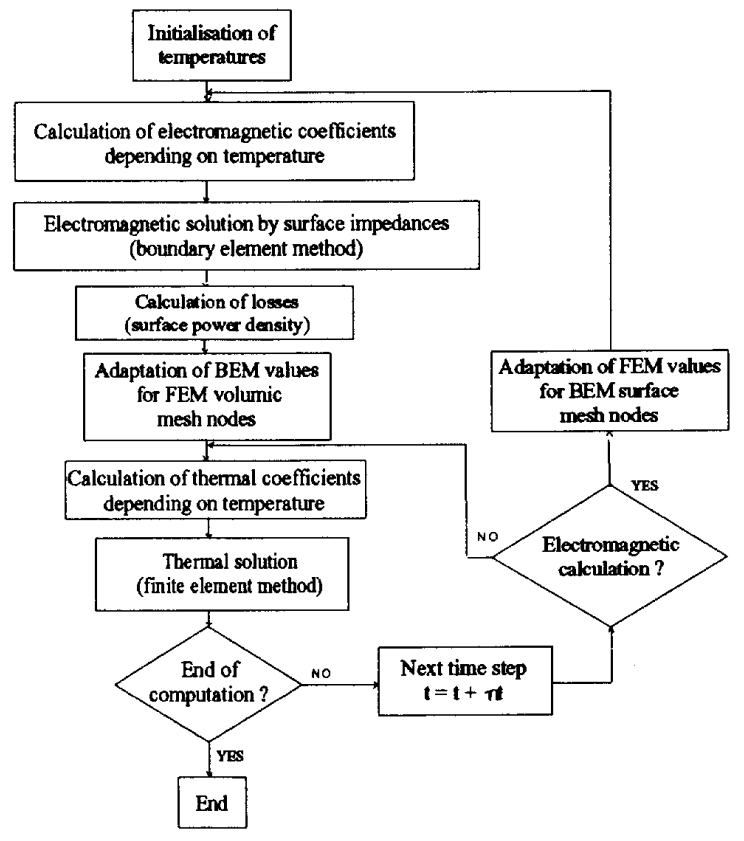

Fig.1. Solution algorithm. 
Because of the strong non-linearities, especially at the Curie-temperature $\left(\mathrm{T}_{c}=760^{\circ} \mathrm{C}\right)$, we couple by iterations, called weak or indirect coupling [5]. At each time step the result is checked for convergence. Then a decision is made to continue or terminate the computation. This coupling assures a reasonable compromise between the accuracy of the results and the time of computation as well as memory space.

We couple two completely different software packages working separately, which would not be possible by a direct coupling. The meshing of the magnetic problem is only defined on the electrically active parts of the piece (BEM). For the thermal problem only the interior of the piece is meshed (FEM). Thus the calculated values of one meshing must be interpolated on the points of the other meshing and vice versa.

\section{APPLICATION}

The described coupling method has been developed for induction hardening problems. The proposed method is applied to the test problem shown in Fig. 2.

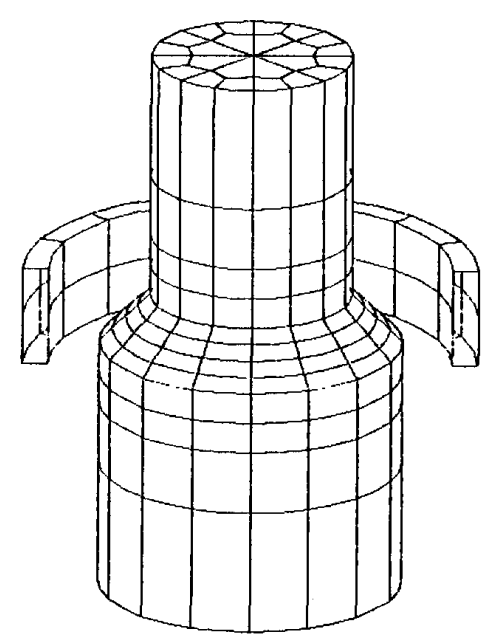

Fig.2. Test problem.

\section{A. Test parameters}

Load material: Inductor material: Inductor current: Frequency:

Convection coefficient:

Radiation coefficient: steel. copper (solid). $1000 \mathrm{~A}$.

$10 \mathrm{kHz}$.

$10 \mathrm{~W} \mathrm{~m}^{-2} \mathrm{~K}^{-1}$.

$1.5 \mathrm{~W} \mathrm{~m}^{-2} \mathrm{~K}^{-4}$.
Fig. 3 shows the physical properties of the ferromagnetic material which are approximated and used iteratively by the presented coupling method.

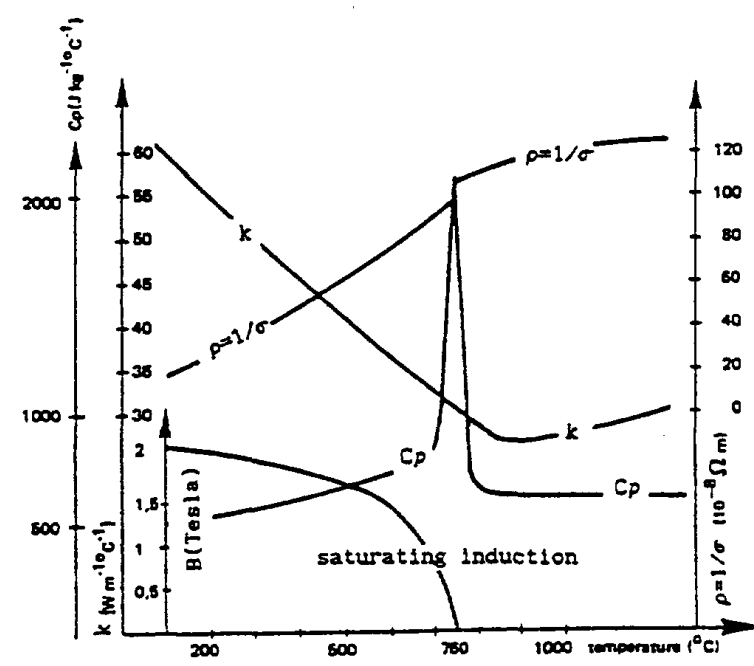

Fig.3. Physical properties.

\section{B. Test cases}

Two cases have been treated. Test 1 is an axisymmetric problem (Fig. 4 and Fig.5). It is a metal barrel with two diameters $(40 \mathrm{~mm}$ and $60 \mathrm{~mm}$ ) and a solenoid inductor orthogonal to the barrel axis.

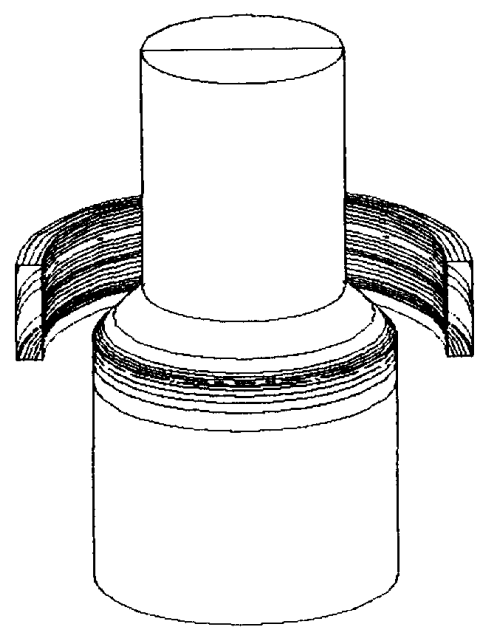

Fig.4. Magnetodynamic result of TEST1.

The results can be compared easily to those obtained by 2D magneto-thermal packages already used in industry. Test 2 consists of the same barrel as TEST 1 but the inductor is no longer centered. It has been moved $2 \mathrm{~mm}$ orthogonally to 
the barrel axis, so the problem is not axisymmetric (Fig.6 and Fig.7).

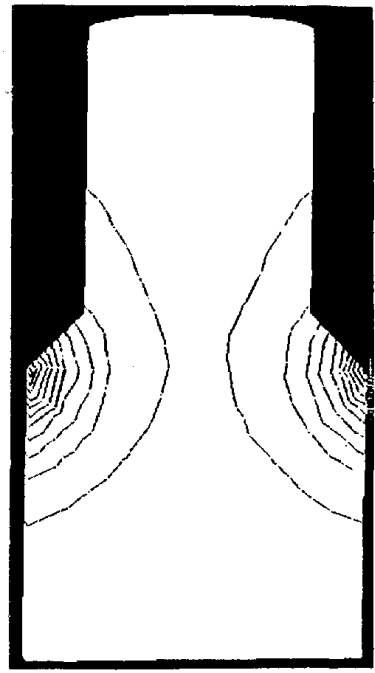

Fig.5. Thermal result of TEST1

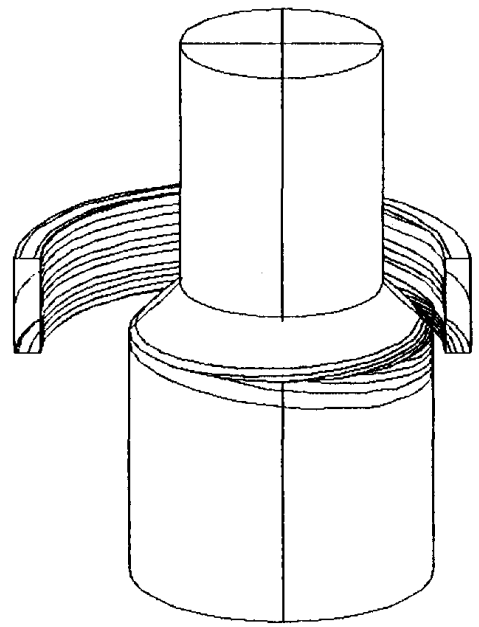

Fig.6. Magnetodynamic result of TEST2.

\section{Results}

Modelling a complete heating process takes much CPUtime and usually much inter action. To reduce the user's operating time a supervisor package has been developed to carry out the automatic data transfer and the iterative process control. Fig. 4 to Fig. 7 show the influence of the distance between the inductor and the load on the form and position of the heated areas. Also the power density values are higher where the inductor is situated nearer to the load.

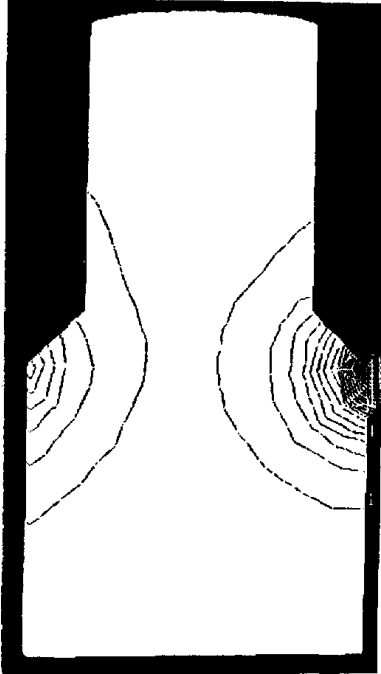

Fig. 7. Thermal result of TEST2.

\section{CONCLUSION}

The presented magneto-thermal coupling method is well adapted to 3 dimensional induction hardening problems with complex shapes. The results for the presented test problem are close to those obtained by calculating a cross-section with an established 2D magneto-thermal package. Slite differences are evident because the electromagnetic part of the problem is calculated with linear physical properties.

\section{ACKNOWLEDGMENT}

The authors would like to thank Mr. Jacques BOULLE of Renault S.A., Industrial Systems Engineering Department in Paris, France for his support of this work and Mr. Xavier Brunotte of Cedrat Recherche in Grenoble for his help concerning the volumic meshing by means of FLUX3D.

\section{REFERENCES}

[1] PH. MASSE, "Modelling of continuous media methodology and computer-aided design of finite element programs. IEEE Trans. Magn., vol. 20 , no. S, pp.1885-1890, 9/1984.

[2] A BOSSAVIT, "Modèles et modèlisation en électrotechnique," Techniques de l'Ingenieur, A 1207.

[3] F. BOUILLAULT, "Formulations magnétiques 3D pour la résolution des équations de la magnétodynamique," RGE, No.2/92, Fév.1992.

[4] L. KRÄHENBÖHL, D. MULLER, "Thin layers in electrical engineering Example of shell models in analysing eddy currents by boundary and finite element methods," IEEE Trans. Magn., vol. 29, no. 2, 3/1993.

[5] M. FELIACHI, G. DEVELEY, "Magneto-thermal behavior finite element analysis for ferromagnetic materials in induction heating devices," IEEE Trans. Mag., Vol. 27, no. 6, 11/1991. 